\title{
Interpolation operators on a triangle with two and three curved edges
}

\author{
AlinA BABOŞ
}

\section{ABSTRACT.}

We construct Lagrange, Hermite and Birkhoff-type operators, which interpolate a given function and some of its derivatives on the border of a triangle with two and three curved edges. We also consider some of their product and boolean sum operators. We study the interpolation properties and the degree of exactness of the constructed operators.

\section{REFERENCES}

[1] Baboş, A., Some interpolation operators on triangle, The 16th International Conference The Knowledge - Based Organization, Applied Technical Sciences and Advanced Military Technologies, Conference Proceedings 3, Sibiu, 28-34, (2010)

[2] Baboş, A., Some interpolation schemes on a triangle with one curved side, General Mathematics (accepted)

[3] Barnhil, R. E., Gregory, J. A., Polynomial interpolation to boundary data on triangles, Math. Comput., 29 (1975), No. 131, 726-735

[4] Barnhil, R. E., Birkoff, G.and Gordon, W. J., Smooth interpolation in triangle, J. Approx. Theory., 8 (1973), 114-128

[5] Barnhil, R. E. and Mansfield, L., Error bounds for smooth interpolation, J. Approx. Theory., 11 (1974),306-318

[6] Bărbosu, D. and Zelina, I., About some interpolation formulas over triangles, Rev. Anal. Numer. Theor. Approx., 2 (1999), 117-123

[7] Bărbosu, D., Aproximarea funcțiilor de mai multe variabile prin sume booleene de operatori liniari de tip interpolator, Ed. Risoprint, Cluj-Napoca, (2002)

[8] Bernardi, C., Optimal finite-element interpolation on curved domains, SIAM J. Numer. Anal., 26 (1989), $1212-1240$

[9] Birkhoff, G., Interpolation to boundary data in triangles, J. Math. Anal. Appl., 42 (1973), 474-484

[10] Blaga, P., Cătinaş, T. and Coman, Gh., Bernstein-type operators on triangle with all curved sides, Appl. Math. Comput., 218 (2011), No. 7, $3072-3082$

[11] Cătinaş, T. and Coman, Gh., Some interpolation operators on a simplex domain, Studia Univ. Babeş Bolyai, LII (2007), No. 3, 25-34

[12] Coman, Gh. and Cătinaş, T., Interpolation operators on a triangle with one curved side, BIT Numer Math., 47 (2010)

[13] Coman, Gh. and Cătinaş, T., Interpolation operators on a tetrahedron with three curved sides, Calcolo, (2010)

[14] Gordon, W. J. and Hall, Ch., Transfinite element methods: blending-function interpolation over arbitrary curved element domains, Numer. Math., 21 (1973), 109-129

[15] Marshall, J. A. and McLeod, R., Curved elements in the finite element method, Conference on Numer. Sol. Diff. Eq., Lectures Notes in Math., Springer Verlag, 363 (1974), 89-104

DePARTMENT OF TECHNiCAL SCIENCES

"Nicolae BALCESCU" LAND ForCES ACADEMY

3-5 Revolutiei Street, 550170, Sibiu, Romania

E-mail address: alina_babos_24@yahoo.com

* Dedicated to Professor Emeritus Constantin Corduneanu on the occasion of his 85th birthday

Received: 09.10.2013; In revised form: 16.11.2013; Accepted: 19.11.2013

2010 Mathematics Subject Classification. 41A05, 41A80.

Key words and phrases. Triangle, curved edges, interpolation operators. 